\title{
Complex fibers orientation distribution evaluation in short glass fiber-reinforced thermoplastic (PA66 GF50)
}

\author{
Prashanth Santharam ${ }^{1,2}$, Thomas Parenteau ${ }^{1,2}$, Pierre Charrier ${ }^{1}$, Denis Taveau ${ }^{1}$, Vincent Le Saux ${ }^{2}$, and Yann Marco $^{2}$ \\ ${ }^{1}$ Vibracoustic, CAE and Durability Prediction Department, 44474 Carquefou, France \\ ${ }^{2}$ Institut de Recherche Dupuy de Lôme (IRDL), UMR CNRS 6027, ENSTA Bretagne, France
}

\begin{abstract}
The use of composites made of polyamide 6.6 matrix and short-glass-fibers in automobile industry is progressive due to its low density and cost. The injection molded short glass fiber-reinforced (SGFR) thermoplastics structural parts such as intake, manifold and engine mount housing, induce complex fiber orientation distributions (FOD). This microstructure governs the macroscopic properties such as the mechanical stiffness and fatigue resistance. To estimate the FOD on such industrial parts at complex angles and ribs, we rely on simulation results and micro tomography analysis. The interest of this paper is to develop a semi-automated, quick and efficient orientation tensor identification approach from 2D microscopic images, which is capable of observing relatively larger surface compared to micro tomography. We finally conclude by comparing it with micro tomography and simulation results. Furthermore, we investigate its relevance with fatigue service ability.
\end{abstract}

\section{Introduction}

The urge to replace heavy metallic parts had made the car manufacturers look for light weight and environment friendly alternatives. Wherein, short glass fiberreinforced (SGFR) thermoplastics fit the exact requirements. They are cost efficient solutions, which combine sufficient stiffness for many structural components and large freedom of shapes provided by injection molding. During the process of injection, the SGFR exhibits specific microstructure. It is characterized by a heterogeneous orientation of the fibers (that leads to an anisotropic mechanical behavior) along the structural part, and also in thickness due to the well-known skin-core effect [1-3]. Due to the macroscopic behavior impact, mechanical response and fatigue resistance of the part are affected to a considerable extent [1]. Currently, the FOD predicted by commercial simulation software are used by the companies in their design loop to improve their parts. The difference between the reality seen on the parts and these simulation results are questionable, thereby creating a demand to clarify the results with experimental analysis. In some cases, even microtomography analysis is seen as a replacement. The evaluation of these tensors is clearly a key point for the design loop, representative with the geometric details met on industrial parts. In order to provide a better or faster description, this paper presents a semi-automated approach, which could be used to analyze large areas of the sample. This 2D microscopic images stores less space than that of tomography analysis. Similarly, the time taken to process the images is also significantly lower. First, the experimental protocols and the image computing procedures are detailed and then a comparison to micro-tomography result is achieved on a simple case. Then, the analysis is extended to describe the orientation tensors on a wider area, and for more complex orientation on structural samples defined previously [1]. Moreover, the identification of additional features like cracks, porosities and clusters of fibers are also presented. Finally, the orientation tensors identified are compared on one hand, to the ones predicted by Moldflow and on the other hand, to the thermal fields and crack locations observed during fatigue tests.

\section{Experiments}

\subsection{Material and samples}

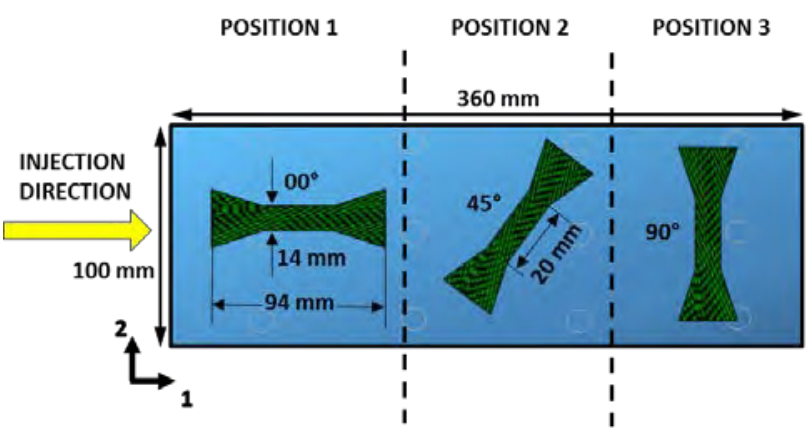

Fig. 1. Injection molding of Dog bone samples.

The material investigated here is a thermoplastic, reinforced with $50 \%$ in mass glass fibers (PA66 GF50) 
provided by Solvay company. The samples were tested at an ambient temperature in a conditioned state (RH50).Two types of samples were used in this study. The first one is called the Dog-bone specimen and is quite standard even if here, we use the Solvay geometry. The Dog-bone specimens are cut from the $\sim 3 \mathrm{~mm}$ thick injection plates as shown in Fig.1. These plates have homogenous and distinct layers though the thickness. For this reason, it's a suitable sample to perform one dimensional analysis of the orientation tensor. It's also necessary to keep in mind that the location (position 1, 2 or 3 shown in Fig. 1) of the sample within the plate has an influence on the orientation tensor evolution at the skin [1]. The orientation of the cut allows for varying the tensile direction versus the main injection direction. In the following, only 0 degree/position 2 samples will be presented.

The second kind of sample is much more specific and is defined to be representative of some geometric details (and therefore injection flows) met on industrial parts. The geometry of the samples called T-Bone is presented in Fig. 2. For this same geometry, two injection locations are possible, providing a very interesting way to modify the obtained microstructure, especially at the flow bifurcation. The first gate location is on the side and is presented in Fig. 2, referenced as T-Bone 1B. The second gate location is from the tip of the central rib and is referenced as T-Bone $1 \mathrm{~A}$.

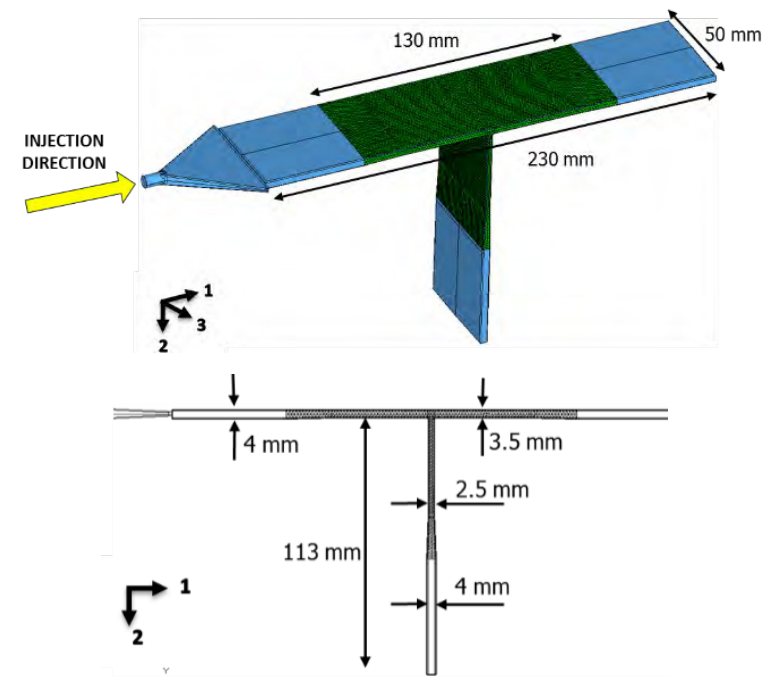

Fig.2 Injection molding of T bone 1B samples.

\subsection{Microstructural observations}

\subsubsection{Optical observation}

The Keyence VHX5000 microscope was used to perform 2D micrograph analysis. The samples are cut to the required location and angle, after which they could be grouped together in a resin (to be time efficient) for the polishing protocol. The resin samples undergo the polish protocol, starting from larger grain size to smaller ones; with polish time ranging from $4 \mathrm{~min}$ to $20 \mathrm{~min}$. The images taken after every polish step are shown in Fig. 3. The edges of the fibers should be preserved with care, as they are necessary to identify their full length. The polish protocol should be enough to distinguish the fibers and matrix as shown in Fig. 3 after the final $3 \mu \mathrm{m}$ polish.

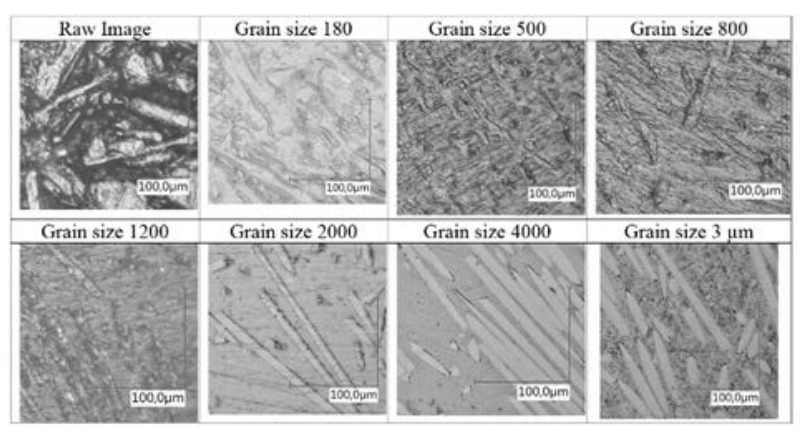

Fig. 3. Specimen surfaces after the various steps of the polishing protocol.

After the polish step, the Keyence microscope is used to take the microscopic 2D images of the sample. Fig. 4 shows the setup of the Keyence microscope observing the samples fixed in the resin. In addition, Fig. 4 indicates the location on the corresponding specimen. Since it is not possible to observe a representative surface with one image, the Keyence image stitching option is used to get a larger view of the specimen. External tools can also be used to serve the purpose [6].

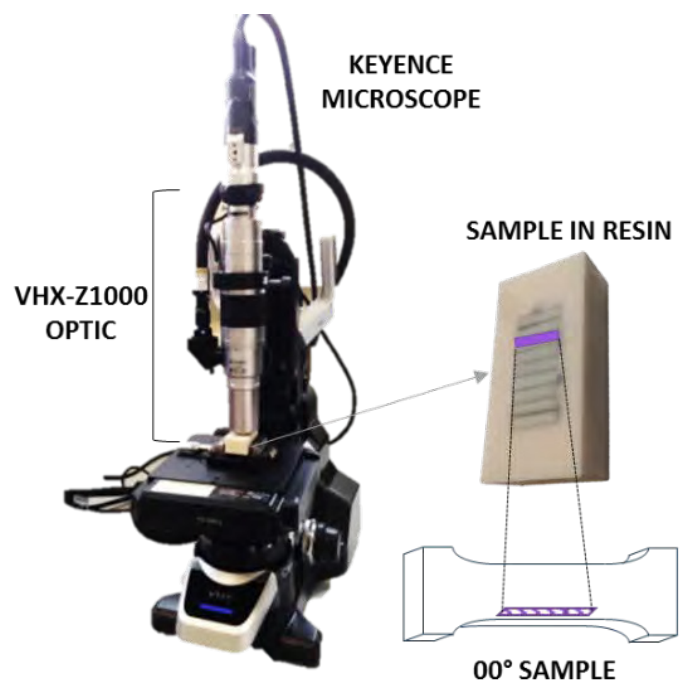

Fig. 4. Polished sample with Keyence microscope.

\subsection{2 $\mu$-Tomography Observation}

The CT scan is used to perform micro tomography analysis on the same material, 0 degree position 2, to obtain orientation tensor evolution along the thickness of the sample. Target is to illustrate and quantify the skincore effect of such samples. The calculation of the orientation tensor and its components are well described in [1-3], [7-10]. For simplicity purpose, only the two components of the second order orientation tensor (a11 and a22) are plotted in Fig. 2 [1], wherein direction 1 represents the injection direction and 2 is the one perpendicular to the injection direction as shown in Fig.1. The motivation of presenting this data is to get 
reliable data that can be compared to the outputs of our image analysis.

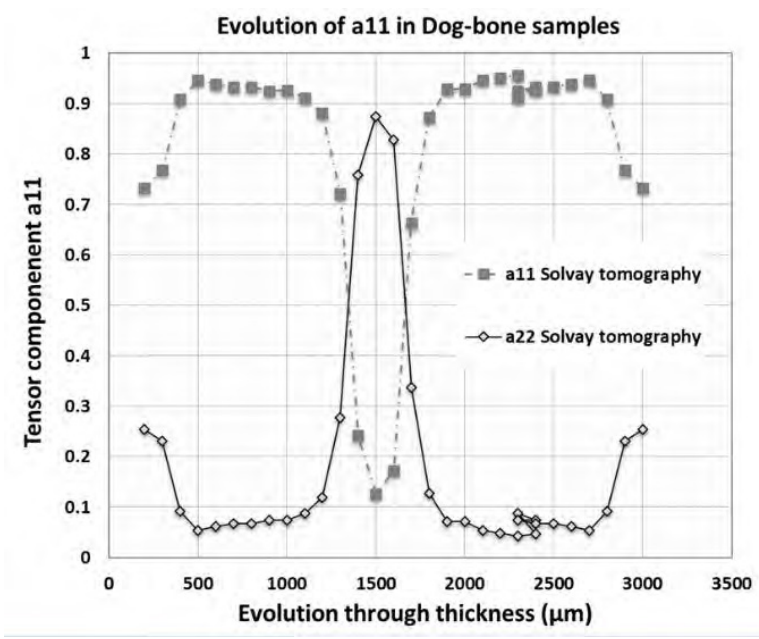

Fig. 5. $\mu$ tomography Dog-Bone orientation tensor

\subsection{Mechanical Test on T-Bone 1B}

The T-Bone 1B sample has been tested under cyclic flexural conditions. The test was displacement controlled, with load ratio and the test frequency as $\mathrm{R}=0$ and $1 \mathrm{~Hz}$ respectively. The details of these tests can be found in $[1,4]$. From experience gained from previous experimental observations, the dissipation fields of the T-bone $1 \mathrm{~B}$ sample indicated that the primary location of failure originates on the right arm (opposite to the injection direction) of the sample almost at the mid-point along the specimen as shown in Fig. 6 [1]. Fig. 6 shows the initial thermal field and the dissipated energy in the region of interest (dashed red box in Fig. 6). During these tests, the thermal fields were recorded by an infrared camera (FLIR SC7600-BB) using a 50mm lens. The thermal resolution is improved with a pixel-wise calibration, taking into account the housing temperature [4]. The calculation of dissipation fields are discussed more clearly in $[1,4]$.

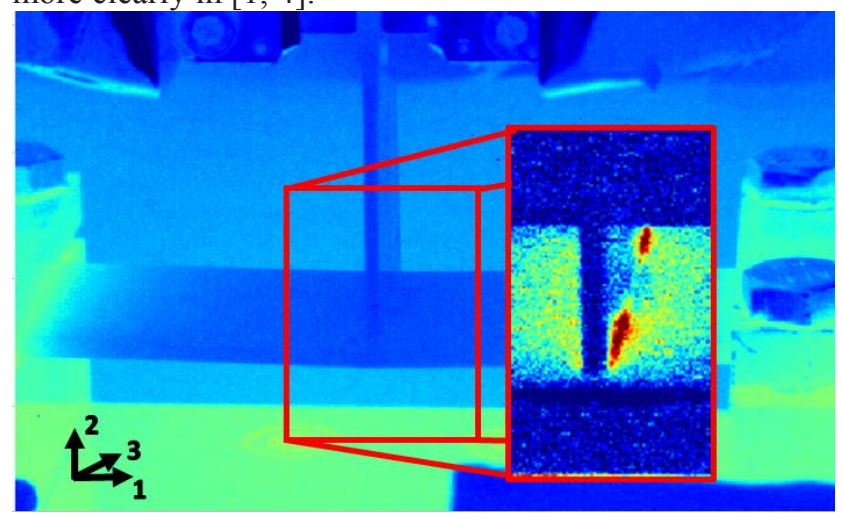

Fig. 6. Thermal field and dissipated energy of T-bone 1B

In order to focus on the location of interest, the Tbone 1B sample was cut into two halves as shown in Fig. 7 (left). During the test, the central rib is fixed in place while the left and right arms are moved simultaneously with the help of an experimental set up shown in Fig. 7.

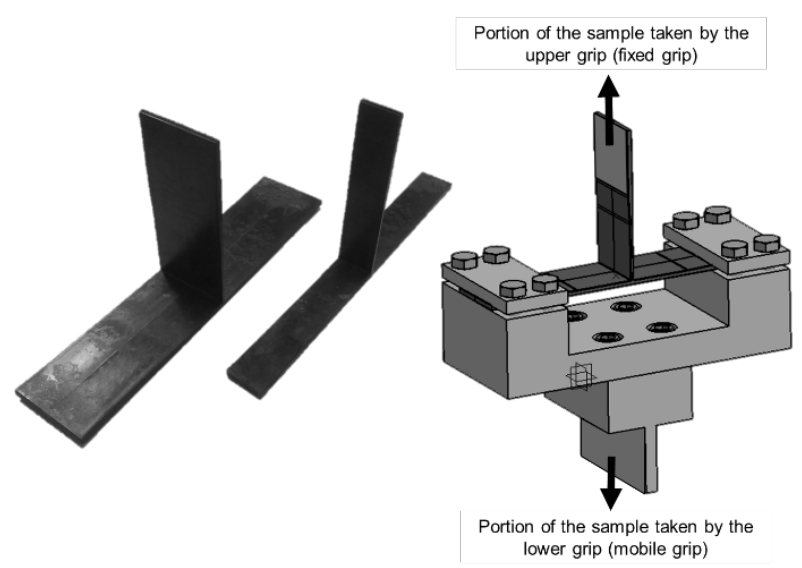

Fig. 7. Half and full T-Bone 1B sample with testing apparatus

\section{Tools for microscopic analysis}

\subsection{Measurement of Orientation Tensor on Dog- bone samples}

The orientation tensor is a second order tensor used to describe the orientation of fibers in composites. Based on the requirements, element based average values of these tensors are used for analysis. Each fiber can be represented by a unit vector $\underline{p}$ carried by its principal axis. It is thus possible to define the orientation of a fiber by the projection of this vector in a global coordinate system (Fig. 9). The components of this vector can be evaluated thanks to the angles $(\theta, \varphi)[1-3],[6,9]$.

$$
\underline{p}=\left\{\begin{array}{c}
p_{1}=\sin \theta \cos \phi \\
p_{2}=\sin \theta \sin \phi \\
p_{3}=\cos \theta
\end{array}\right.
$$

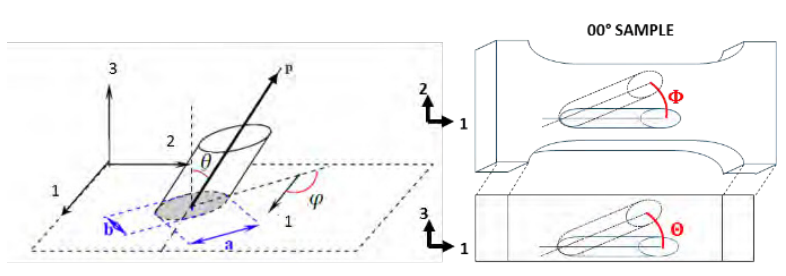

Fig. 8. Orientation tensor calculation [2]

The second order tensor orientation of a fiber $\mathrm{k}$ is determined as the double tensor product of $\mathrm{p}$ as given by equation (2) [1-3], [9].

$\underline{\underline{a}}$ $=\left(\begin{array}{ccc}\sin ^{2} \theta^{k} \cos ^{2} \phi^{k} & \sin ^{2} \theta^{k} \sin \phi^{k} \cos \phi^{k} & \sin \theta^{k} \cos \theta^{k} \cos \phi^{k} \\ \sin ^{2} \theta^{k} \sin \phi^{k} \cos \phi^{k} & \sin ^{2} \theta^{k} \sin ^{2} \phi^{k} & \sin \theta^{k} \cos \theta^{k} \sin \phi^{k} \\ \sin \theta^{k} \cos \theta^{k} \cos \phi^{k} & \sin \theta^{k} \cos \theta^{k} \sin \phi^{k} & \cos ^{2} \theta^{k}\end{array}\right)$ (2)

In order to define this tensor by optical analysis (i.e. by observing the surface), initially, we detect every fibers in the image by identifying the ellipse formed by the cutting plane and the fiber as shown in Fig. 8. Algorithms such as diffusion and water-shed were used 
in the process. Each ellipse is characterized by its two axes, ' $a$ '- the major and ' $b$ '- the minor as shown in Fig. 8 $[4,5]$. The angle $\theta$ is defined by equation (3) $[2,9,10]$.

$$
\theta=\cos ^{-1}\left(\frac{b}{a}\right)
$$

The angle between the axis 1 and the major axis of the ellipse shown in Fig. 8 [2], corresponds to $\varphi$ angle and it depends on the second-moments of the ellipse. The second order orientation tensor can thus be determined for a fiber. To reconstruct the global tensor orientation, it's also necessary to take into account that the probability of cutting a fiber perpendicular to the surface which depends of the length-' 1 ' of the considered fiber, its diameter-'d' and its orientation. This function $F^{k}$ is defined in [2], [7-10].

\subsection{Measurement of Orientation Tensor on Dog Bone samples}

Now that the orientation tensor is calculated for every fiber in the given image, the next step would be to group the fibers. Based on the aspect ratio of the given image, it is subdivided into bins [6]. For example if the given image has equal number of pixels in $\mathrm{X}$ and $\mathrm{Y}$ directions, then the number of bins can also be equal in $\mathrm{X}$ and $\mathrm{Y}$ directions (columns and rows). Fig. 9 is an illustration for the image with bins, it also explains that the fibers are classified based on the centroid location of its bounding box. For the example given in Fig. 9, the fiber is related to grid $(2,2)$ since the centroid of the fiber is in it. The tensor value of every grid is calculated by a simple average of all the fibers (its centroids) located in the grid.

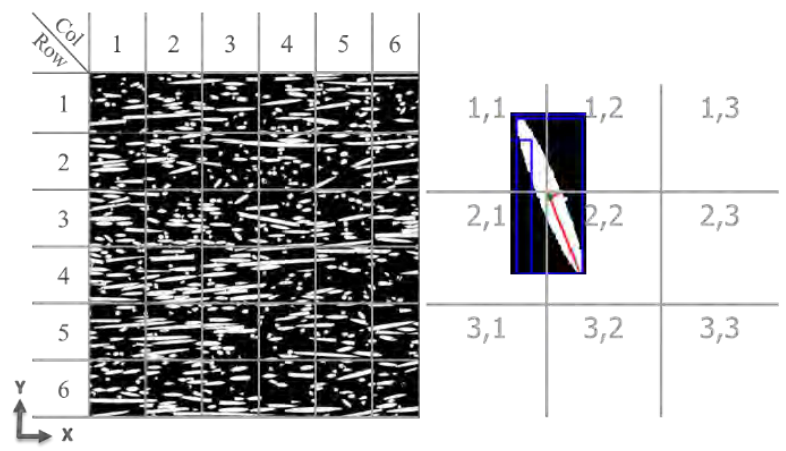

Fig. 9. Classification of fibers using grids

The Fig. 10 is an example of an image (equal pixels in both directions) from 0 degree skin sample position 2 polished from in direction 3 (refer Fig. 1) until the depth of $500 \mu \mathrm{m}$ (approximately in the skin of the sample). Since it's a square image, it was divided into 50 bins in both directions, which are latter plotted as classical histogram. As mentioned earlier in section 1.2, the layers are almost homogeneous, which can be clarified by column wise average tensor value plot shown in Fig. 10. Wherein, the total average value of tensor component $a_{11}=0.89$.

Similarly, on viewing the sample from direction 2, a scattered view of the tensor through the thickness of the

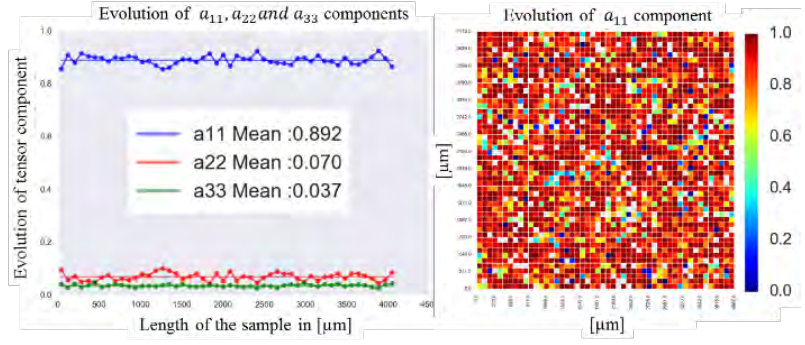

Fig. 10.Tensor Map for top view sample

sample is observed as shown in Fig. 11. The values at the skin are not clearly predicted because the side view (direction 2 view) did not have the good estimation of the tensor component $a_{12}$, which corresponds to the phi $(\phi)$ angle in Fig. 8. For this reason, the phi $(\phi)$ angles are estimated by the top view (direction 3 view as explained in Fig. 10) to rectify the tensor component obtained from the side view (direction 2 view). Which is basically reconstructing the tensor components, considering the phi $(\phi)$ values obtained from the plane 1 , 2 and theta $(\theta)$ obtained from plane 2, 3 (refer Fig. 11). The $a_{11}$ and $a_{22}$ tensor components obtained by this calculation is compared with the orientation tensor components obtained by Solvay [1-3] in Fig. 11. The trend of the evolution is quite comparable except for the values at the skin for both $a_{11}$ and $a_{22}$ components.
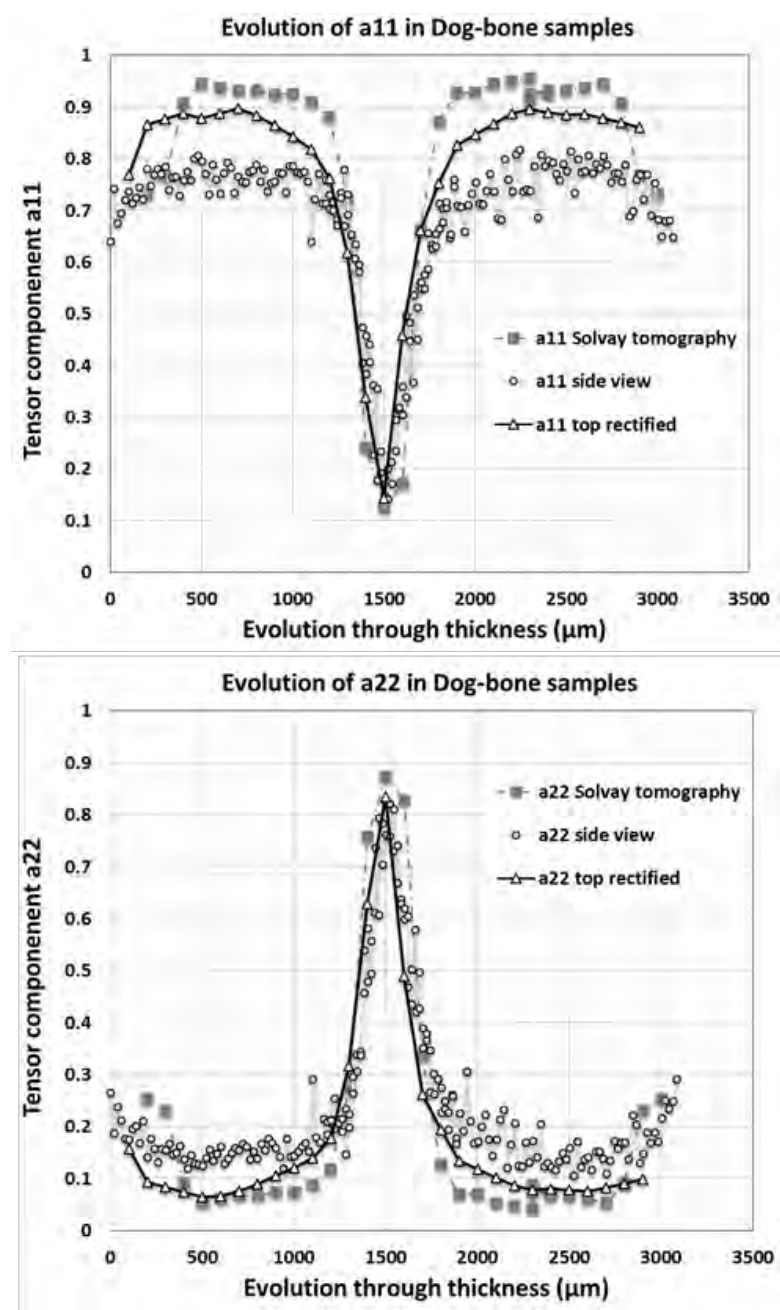

Fig. 11. Evolutions of $a_{11}$ and $a_{22}$ for 0 degree position 2 sample 


\subsection{Measurement of Orientation Tensor on $\mathrm{T}$ bone}

In order to extend the orientation calculation approach on T-bone 1B sample at the region of interest (section 2.3), four microscopic images are obtained from the Keyence, which are combined during the processing step by recognizing the patters in the images [6]. This led to $300 * 200$ bin histogram data, and the $a_{11}$ component of T-bone 1B is the plot is shown in Fig. 12.

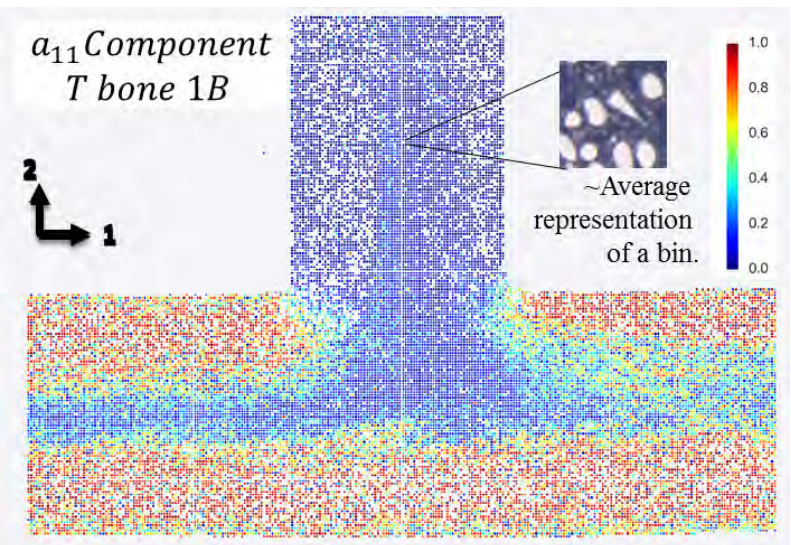

Fig. 12. Orientation tensor map for T-bone 1B sample

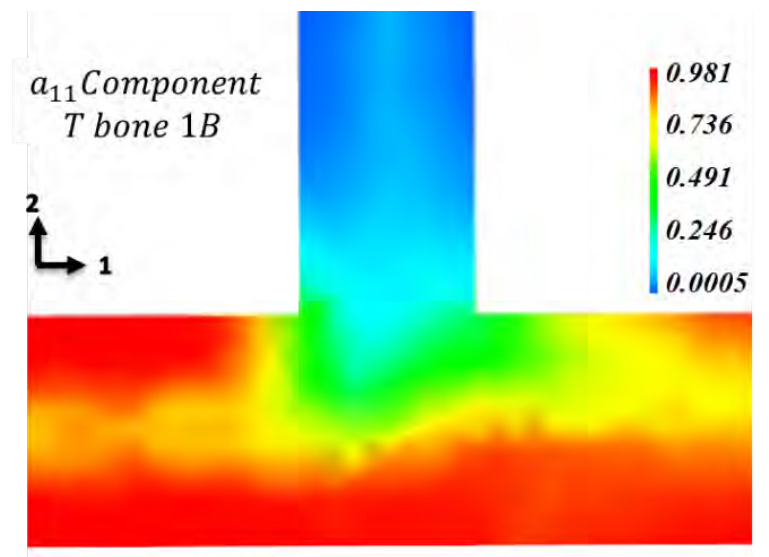

Fig. 13. Orientation tensor for T-bone 1B - Moldflow

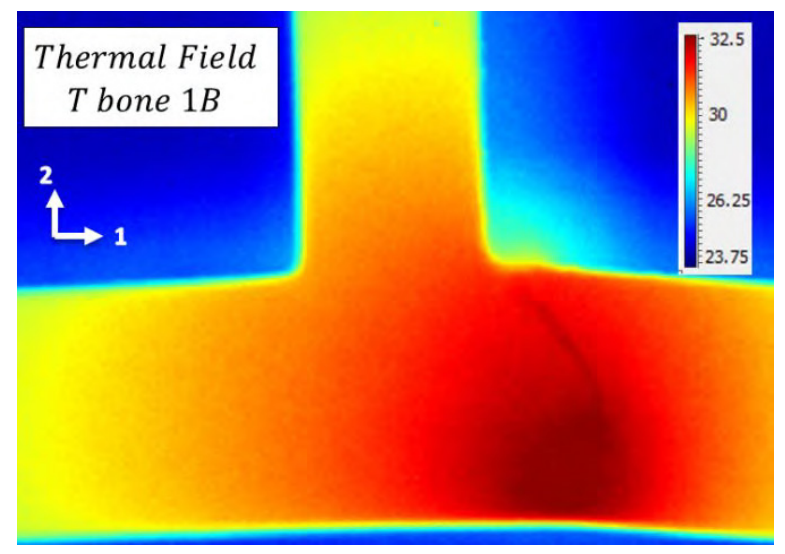

Fig. 14. Thermal field for T-Bone 1B sample

In the figure, the average representation of one bin in the histogram is highlighted. It is possible to notice that there are many empty bins, which means that no fiber were detected or highlights the presence of fiber cluster or void or crack which have replaced the fibers in that location. Detection of data other than fibers are discussed in section 3.3.

\subsection{Measurement of additional features}

When attempts were made to analyze the microscopic images even further, it was possible to distinguish the voids and cracks from the rest of the matrix and fibers based on grey scale as shown in Fig. 15 (grey scale of Keyence Image (A) in Fig. 16) $[5,6]$. The dark pixels in the image were identified either as cracks or voids, therefore the peak at the darker region were identified.

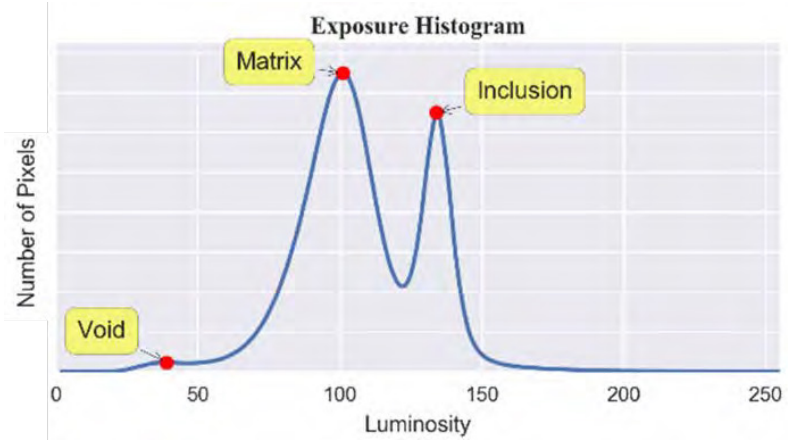

Fig. 15. Grey scale of the total image used for analysis

In Fig. 16, the Keyence Image (A) show a closer view of the T-Bone 1B sample, the Processed Image (A) shows the processed data which has identified the voids as pixels are presented (cracks can also be located). With this information, it is possible to have a good estimation of distribution and size of the voids and cracks. The Tbone 1B sample, many such voids shown in Fig. 16 were clearly located at the flow bifurcation region. For the same sample, when viewed from a different direction as shown in Fig. 17, many clusters and voids are noticed. The clusters are normally identified if the total volume of the detected contour is larger than the maximum possible area of the fiber $[5,6]$. Similar to voids, it is possible to quantify the location, distribution, size in addition the orientation of the clusters.
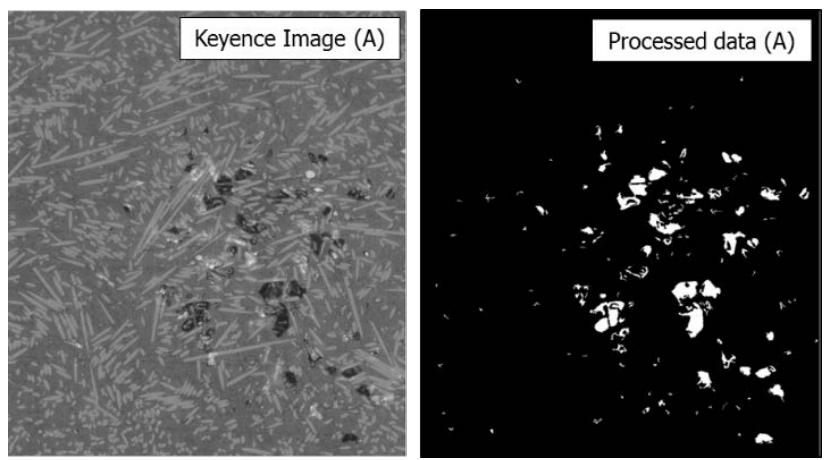

Fig. 16. Keyence images with identified voids and cracks

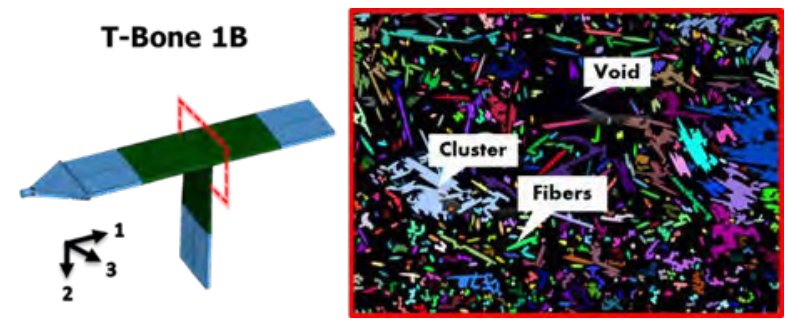

Fig. 17. Clusters, fibers and voids in T-Bone 1B sample 


\section{Comparison to numerical predictions and to fatigue observations}

\subsection{Comparison to the orientation tensor predicted by injection simulation}

The Moldflow simulation results for the same T-bone 1B sample is presented in Fig. 13 [1], wherein the injection is along direction 1. On the first view, the trend of the orientation tensor is quite similar to Fig. 12 obtained by microscope observations. The first remark is that, in both cases, the orientations in the left and right arms are completely different, at least on the regions that are close to the bifurcation. This is of course induced by the unbalanced filling history, as the gate is located on the left arm along direction 1 . The second remark is related to the mesh size and quality, Fig. 13 shows a Moldflow mesh with 12 element layer (through thickness) with 1.7 million elements in full model. This quality of mesh was enough to describe the overall orientation gradient. If we now come specifically to the comparison between the simulations and the microscopic observations, the following points are observed. The skin of the left arm appears to be well oriented in both the cases. Even the aftermath of the bifurcation is clearly visible by change of directions in the orientation tensor. On closer examination, the skin-core effect is more pronounced in the experiment than in the simulation. The skin of the central rib is well described with lower values in the $a_{11}$ component and higher values in $a_{22}$ component. Even the values at the core are comparable. It therefore seems that the injection simulations give a reasonable evaluation of the global shape of the orientation tensor, but fails to identify the local gradients.

\subsection{Comparison to the fatigue observations}

The thermal fields were observed on regular intervals around the region of interest during the fatigue test. The fatigue test followed the test discipline mentioned in section 2.3. Further details of the self-heating tests are mentioned in [1]. For fatigue tests on different loading conditions, the T-bone 1B sample always failed on the right arm (opposite to the injection direction). This was repetitive and even the location of failure was consistent [1]. The orientation tensor could explain this observation, as the combination of the mechanical fields

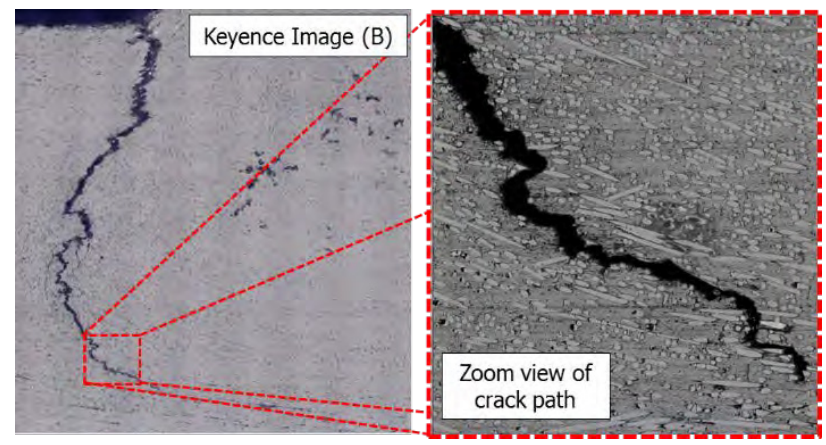

Fig. 18. Crack path observed using Keyence microscope induced by the stress concentration in the vicinity of the radius will lead to different failure consequences as the local orientations are clearly different. This point would require a deeper investigation using Finite Elements simulations. The last point to highlight is that the crack propagation path appears to follow the direction of orientation as seen in the Keyence Image (B) at Fig. 18 for T-bone 1A sample.

\section{Conclusions}

This paper presented a semi-automated approach to determine the SGFR orientation tensors, based on 2D microscopic images and on principles already developed elsewhere. This technique exhibited a faster analysis protocol than the ones using micro-tomography. Moreover, a larger area can be efficiently analyzed thanks to the combination of several observation zones. Beyond the orientation tensor, the developed tools are also giving access to other interesting microstructural features (cracks, porosities and clusters of fibers). Finally, the identified orientation tensors have been compared to the ones predicted by Moldflow and to the thermal fields and crack locations observed during the fatigue tests. It appears that these comparisons provide very precious understanding and validation cases. The perspectives are wide on this topic, from measuring the thermal fields at the microstructure's scale, to finer investigation of the mechanical failure dependency on the microstructure characteristics.

\section{References}

1. L. Serrano, Caractérisation thermomécanique $d u$ comportement en fatigue des thermoplastiques renforcés de fibres de verre courtes, PhD UBO, Brest (2015)

2. A. Megally, Étude et modélisation de l'orientation de fibres dans des thermoplastiques renforcés, $\mathrm{PhD}$ ENSAM, Paris (2005)

3. D. Dray, Prediction of the thermoplastic properties of an injection molded composite, PhD ENSAM, Paris (2008)

4. V. Le Saux, C. Doudard, Infrared Physics and Technology 80, 83-92 (2017)

5. L. Grady, IEEE Trans. Pattern Analysis and Machine Intelligence 28, 1768-1783 (2016)

6. J. Erik Solem, Programming Computer Vision with Python, (2012)

7. R.S. Bay and C.L. Tucker III, Polymer Engineering \& Science, 32(4), 240-253, 317, 332 (1992)

8. C. Eberhardt and A. Clarke, Composites Science and Technology, 61(10), 1389-1400 (2001)

9. S.G. Advani and C.L. Tucker III, Journal of Rheology, 31(8), 751-784 (1987)

10. M. Gupta and K.K Wang, Polymer Composite 33, 410 (1993) 